\title{
Performance modelling of the Darrieus wind turbine
}

\author{
Thuzar Mon ${ }^{1}$, and Supakit Worasinchai ${ }^{2, *}$ \\ ${ }^{1}$ Department of Automotive Engineering, Thailand Advanced Institute of Science and Technology (TAIST), King Mongkut \\ Institute of Technology Lad Krabang, Bangkok, 10520, Thailand \\ ${ }^{2}$ Machinery Development Research Team, Material Processing and Manufacturing Automation Research Group, National Metal \\ and Materials Technology Center, 12120, Thailand
}

\begin{abstract}
Three-dimensional numerical investigation of the Darrieus wind turbines equipped with different aerofoils is presented in this paper. In the modelling, the computational domain was divided into three different domains and they are blade, rotor, and tunnel domains. A cylindrical domain was created to cover the blade area so that a fine mesh can be applied. The Computational Fluid Dynamics (CFD) was employed to solve and analyze the flow field around the turbine. The Menter Shear Stress turbulence model was chosen in this investigation. Boundary conditions applied were velocity at the inlet, pressure opening at the outlet, and symmetry on other sides. Comparison of simulation results and experiments showed good agreement. The investigation of the effects of the rotor solidity and the aerofoil shape was performed. The simulation results reveal that the aerofoil shape has a significant impact on the turbine performance. For the rotor solidity of 0.7 , the change from the NACA section to the S1046 leads to a reduction of power at low tip speed ratios but the performance improvement is observed when the tip speed ratio is greater than 1.5 . With the lower solidity of 0.375 , the effects of the aerofoil change is less pronounced at low tip speed ratios. Nevertheless, the maximum power coefficient increases for both cases. Further analysis shows that the S1046 is less sensitive to the wind speed change and is promising in the urban application where the wind speed is relatively low.
\end{abstract}

Keyword. Darrieus Wind Turbine, Performance modelling, Aerofoil shape, Rotor solidity

\section{Introduction}

As the world has become struggle with the fossil-fuel shortage and the environmental pollution, the renewable sources of energy have become increasing popular and the use of renewable sources to produce electricity is significantly increased. The wind energy is one of the sources and its capacity of installation has increased significantly in the last decade [1].

Wind turbines have been employed for decades to generate electricity. Its technology development leads to large-scale clean electricity production. Apart from the large-scale production, the idea of decentralizing of the energy sources is beneficial as it can provide power to the specific areas and increase the amount of the overall installed capacity. This leads to a wide interest in the vertical axis wind turbines for the small-scale power generation [2].

The vertical axis wind turbines have a number of advantages over the horizontal axis counterpart as it is insensitive to wind direction and needs no yaw mechanism [3]. Nevertheless, one of its drawback is that it has a relatively poor starting performance and its blade design needs further investigation to improve this performance [4].

Beri and Yao [5] had studied the self-starting performance of the Darrieus wind turbines. The study showed that the employment of asymmetrical aerofoils leads to an improved self-starting behavior with a reduction in peak efficiency. Mohamed et al. [6] further numerically investigated the effect of the aerofoil shape and it had been found that the LS(1)-0413 aerofoil gives maximum power coefficient of 0.415 with an increase around $10 \%$ compared to the conventional NACA 0018 section.

Many studies had investigated effects of the aerofoil thickness on the turbine performance [7-8]. Castelli and Benini [7] numerically evaluated the performance of the Darrieus turbine equipped with NACA0012 and NACA0021. Takahashi et al [8] carried out numerical simulations and wind tunnel experiments to study the effects of the aerofoil shape. The results showed that the NACA0024 that has a high value of thickness is the best aerofoil. Both studies indicated that, for the conventional NACA sections, the increase of the aerofoil thickness could improve the turbine performance.

A series of newly designed, low-speed aerofoils had been investigated for the Darrieus wind turbine [9-10]. Fang et al [9] investigated the employment of S-series aerofoils on the straight-bladed vertical axis wind turbine in comparison with the conventional aerofoil sections such as the S809 and the NACA0018. The results showed that the S1046 aerofoil section performs better than the conventional sections, implying that a good selection of the aerofoil shape can significantly improve the performance of the turbine. Hshem et al [10] investigated the aerodynamic performance of H-rotor wind turbine by using 24 different symmetric and non-

* Corresponding author: supakit.wor@mtec.or.th 
symmetric aerofoils. It was found that S1046 aerofoil show the maximum power coefficient of 0.3463 which performs the best of the tip speed ratio of 2 to 7 . This work presents a numerical investigation of the effects of the aerofoil shapes and the rotor configuration on the performance of the Darrieus wind turbine.

\section{The turbine model}

The turbine models investigated in this paper is threebladed turbines with different rotor configurations. The first model is square and has the same width and height of 0.8 metre. The second model is rectangle and has the width and the height of 1.6 and 0.8 metre, respectively. Both models are investigated when they are equipped with two different aerofoils, which are the NACA0021 and the S1046. Both rotors have the same blade chord of 0.2 metre, leading to a solidity of 0.75 and 0.375 , respectively. The geometry of two turbine models is summarised in Table 1.

Table 1. Turbine configurations

\begin{tabular}{|l|l|l|}
\hline Geometry & Turbine 1 & Turbine 2 \\
\hline Aerofoil section & $\begin{array}{l}\text { NACA0021/S104 } \\
6\end{array}$ & NACA0021/S1046 \\
\hline $\begin{array}{l}\text { Number of } \\
\text { blades }\end{array}$ & 3 & 3 \\
\hline Chord length & $0.2 \mathrm{~m}$ & $0.2 \mathrm{~m}$ \\
\hline $\begin{array}{l}\text { Rotor diameter, } \\
\text { D }\end{array}$ & $0.8 \mathrm{~m}$ & $1.6 \mathrm{~m}$ \\
\hline Blade span, H & $0.8 \mathrm{~m}$ & $0.8 \mathrm{~m}$ \\
\hline Solidity & 0.75 & 0.375 \\
\hline
\end{tabular}

The NACA0021 aerofoil is a thick section with $21 \%$ thickness at the $30 \%$ of the chord. It possesses soft stall behaviour with a moderate maximum lift coefficient. It is commonly employed on low speed wind turbines and hydropower applications [11]. It is reported that the use of the NACA0021 leads to a relatively high performance, compared to other aerofoils with comparable thickness such as the DU91-W2-250 aerofoil [12]. The S1046 aerofoil is a symmetrical section with $17 \%$ thickness at $30.7 \%$ chord. It was designed for low wind speed application and might be promising for urban wind turbine applications. Mohamed et al [6] mentioned that the S1046 is a very promising aerofoil shape for wind energy generation compared to the other symmetric aerofoils.

\section{Numerical methodology}

\subsection{Numerical setup}

The three dimensional numerical analysis of the straight bladed Darrieus wind turbine is conducted by using the computational fluid dynamic (CFD).

The computational domain was divided into three parts and they are wind tunnel region, rotor region, and blade region as shown in Figure 1. Boolean operations were performed to subtract the three cylindrical blade domains from the cylindrical rotor domain and to subtract the cylindrical rotor domain form the outer wind tunnel domain. An interface boundary condition was assigned at the contact surfaces of the rotating domains and the outer fixed domain, as depicted in Figure 1a. The size of each regions were configured based upon the rotor diameter [13] as presented in Figure $1 \mathrm{~b}$ and they are summarised in Table 2.
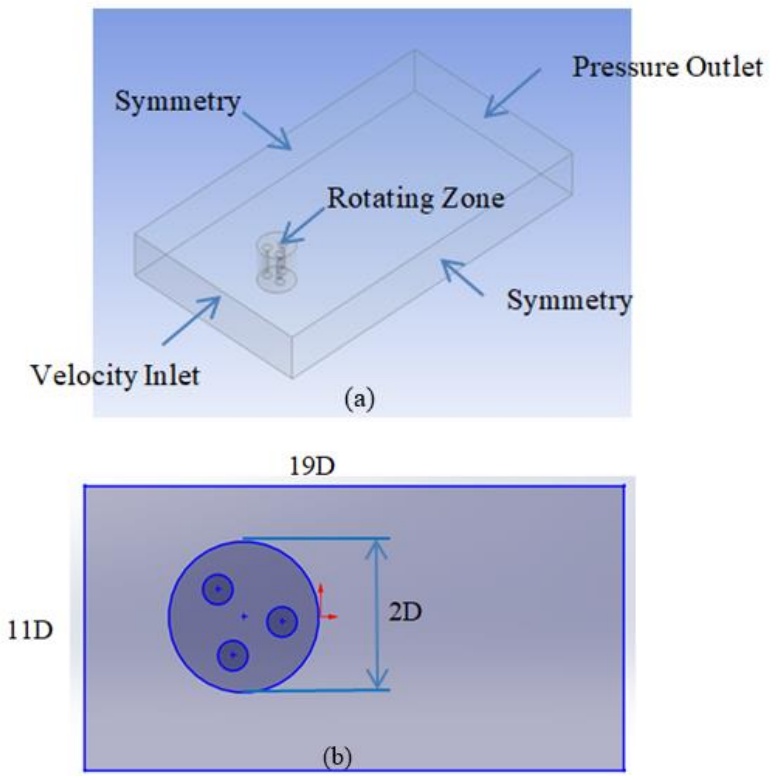

Fig. 1. (a) Boundary conditions (b) Plane view of three domains with specified dimensions

Table 2. Specified dimensions of CFD domain

\begin{tabular}{|l|l|l|}
\hline Domain & Denomination & Value \\
\hline \multirow{3}{*}{ Wind tunnel } & Length & $19 \mathrm{D}$ \\
\cline { 2 - 3 } & Width & $11 \mathrm{D}$ \\
\cline { 2 - 3 } & Height & $2 \mathrm{D}$ \\
\hline \multirow{2}{*}{ Rotor } & Diameter & $2 \mathrm{D}$ \\
\cline { 2 - 3 } & Height & $1.85 \mathrm{D}$ \\
\hline Blade & Diameter & $0.5 \mathrm{D}$ \\
\cline { 2 - 3 } & Height & $1.5 \mathrm{D}$ \\
\hline
\end{tabular}

\subsection{Numerical validation}

The present simulation results were validated against the experimental measurement investigated by Elkhoury et al. [13].

\subsubsection{Mesh and time step study}

Mesh quality has an important influence in obtaining accurate CFD results. The most important factor to identify a good grid resolution is the ability to predict the time and place of flow separation around the turbine blades [14]. This prediction is the most important factor in the overall performance of turbine at low tip speed ratios. The characteristic cell size on both sides of the interface (rotor sub-grid and fix sub-grid areas and rotor 
sub-grid and blades sub-domains) has the same characteristic in order to gain faster convergence [13].

In order to ensure that the solution is independent of the mesh size, numerical simulations were conducted using three different mesh element number: 2717718, 3623942 and 4044312 , which were defined as coarse, medium, and fine. The boundary layers of 15 and the surfaces growth rate of 1.2 were applied to the mesh inflation to ensure a sufficient boundary layer. Mesh quality was checked in terms of its skewness around the blades and the overall domain and the angle of the skewness of the cells 0.9. Mesh independency was made for the NACA 0021 at a wind speed of $8 \mathrm{~m} / \mathrm{s}$ and at TSR 0.25 . Table 3 shows the number of nodes, mesh elements, and the value of the average torque generated by the rotor blades for three cases. Figure 2a presents the overall picture of the final mesh where a coarse mesh was applied outside the rotor domain. The rotor domain in the Figure 2a has three cylindrical domains inside that cover each blade as shown in Figure $2 b$.

Table 3. Effect of Mesh on the torque at the rotor blades

\begin{tabular}{|c|c|c|c|}
\hline & Nodes & Elements & $\begin{array}{c}\text { Average torque } \\
\text { (Nm) }\end{array}$ \\
\hline Coarse & 941749 & 2717718 & 1.9668 \\
\hline Medium & 1188335 & 3623942 & 2.2035 \\
\hline Fine & 1288912 & 4044312 & 2.1764 \\
\hline
\end{tabular}

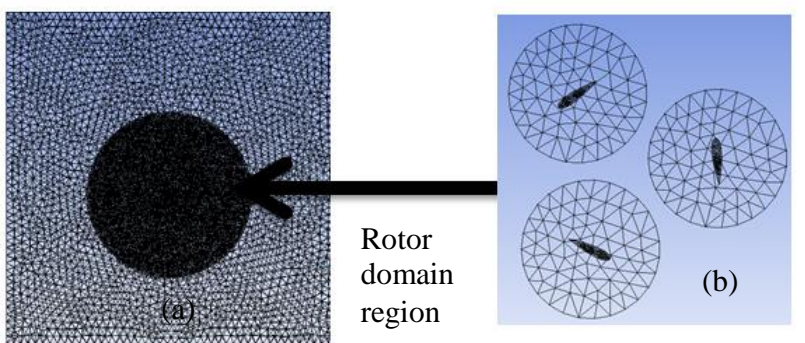

Fig. 2. Mesh near surface clustering of the CFD domain

Simulations results indicated that for mesh element numbers 2717718 and 4044312 shows a difference less than $0.5 \%$. Therefore, in order to obtain the accurate results and to save the computational time, a coarse mesh was set up for the fixed domain. The finest unstructured mesh was used in the rotating domains with specific sizings and inflation layers to be accurate for solving near-wall flow field around the rotor blades.

A suitable time step is another important factor for good CFD simulations. Two different time steps were carried out for the turbine with the NACA0021 at the TSR of 0.25 and at the wind speed of $8 \mathrm{~m} / \mathrm{s}$ with the time steps of 0.00139626 and 0.125664 for one revolution respectively. The power coefficient of 0.020281 , which is much more close to the predicted result, was obtained when the time step is 0.00139626 .

\subsubsection{Turbulence model validation}

Two turbulence models were evaluated to predict the turbine performance: the Large Eddy Simulation (LES) model and the Shear Stress Transport (SST) model.
The Large Eddy Simulation (LES) is a turbulence model focusing on resolving eddies within the flow with an appropriate mesh size. It is based upon Kolmogorov's theory that the large eddies of the flow are dependent on the geometry while the smaller scales are more universal. This implies that large eddies can be solved implicitly and the smaller scales can be solved through subgrid-scale model [15].

The Shear Stress Transport (SST) k- $\omega$ model is a two-equation turbulence model developed by Menter [16]. It is actually a hybrid model that combines the k- $\omega$ formulation in the inner region of the boundary layer and the $\mathrm{k}-\varepsilon$ model in the bulk flow. The validation results are presented in Figure 3.

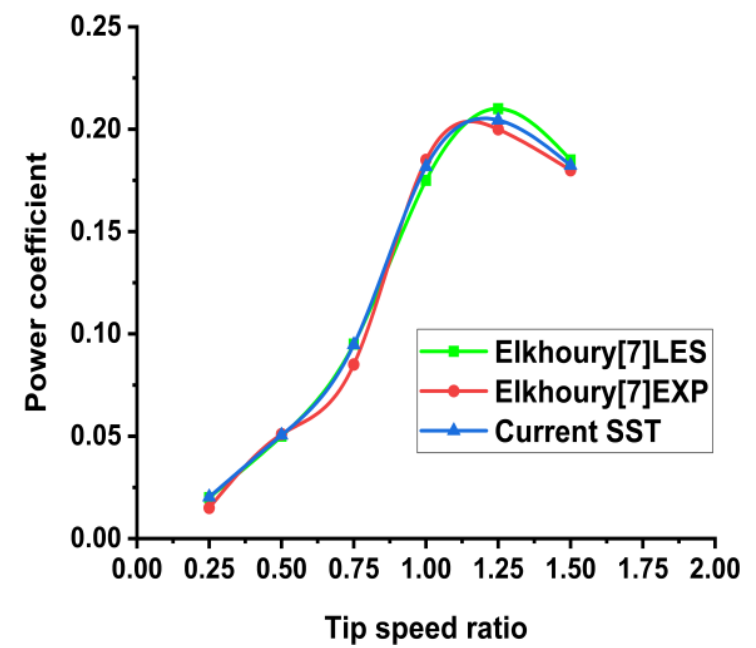

Fig. 3. Power coefficient validation

As can be seen from the Figure 3, the LES and the SST models can predict the experiments well for the whole range of the tip speed ratio. It is worth noting that the discrepancies between numerical and experimental data were calculated in terms of the normalized mean square error (NMSE). The maximum error occurred at the tip speed ratio of 0.25 with values of $8.33 \%$ and $9.16 \%$ for the LES and the SST models, respectively. Nevertheless, this deviation at the peak power output were $0.23 \%$ and $0.048 \%$ for the LES and the SST models, respectively. The SST model was then selected to evaluate the turbine performance in this course of study.

\section{Results and discussion}

The aerodynamic characteristics of H-rotor Darrieus are investigated in terms of power coefficient at different tip speed ratio.

The turbine power coefficient is defined as:

$$
C_{P}=T \omega / \rho R h V^{3}
$$

and as a function of the tip speed ratio, defined as:

$$
T S R=R \omega / V
$$


Where $C_{P}$ is the turbine power coefficient, $T$ is the turbine torque, $\omega$ is the turbine rotational speed, $\rho$ is the air density, $H$ is the blade span length, $R$ is the turbine radius, and $V$ is the inflow velocity.

Figure 4 shows the power coefficient of the rotor with high solidity of 0.75 for two aerofoil sections (the NACA0021 and the S1046) at the wind speed of $8 \mathrm{~m} / \mathrm{s}$. It can be observed that the maximum power coefficient and the operating tip speed ratio depends on the aerofoil shape. The maximum power coefficients are 0.2044 and 0.2103 for the NACA0021 and the S1046, respectively. In addition, while the turbine with the NACA0021 exhibit its maximum power at the tip speed ratio of 1.25 , the turbine with the S1046 exhibits its peak power at a higher tip speed ratio of 1.50 .

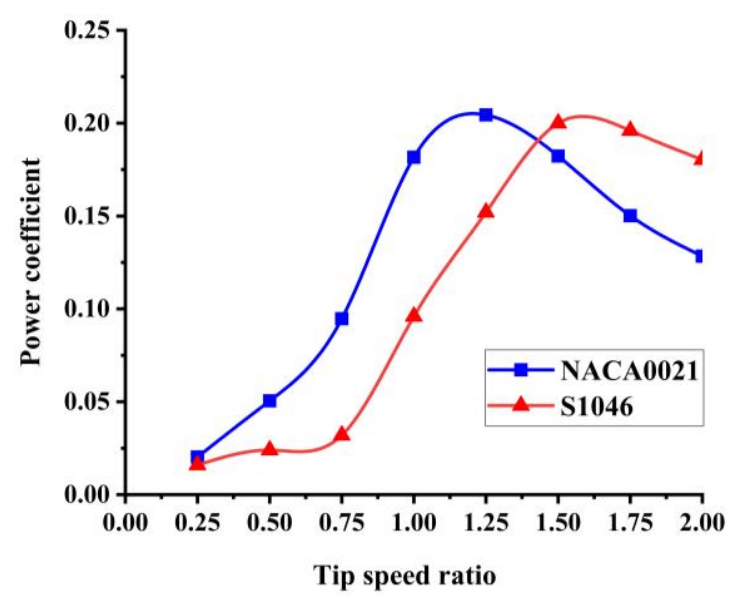

Fig. 4. Power coefficient at a wind speed of $8 \mathrm{~m} / \mathrm{s}$ and the solidity of 0.75

It is worth noting that the power coefficient of the turbine equipped with the NACA0021 outperforms the turbine with the S1046 section at tip speed ratios lower than 1.5 and this is anticipated to be the effect of the thickness that promotes more suction peak at low TSRs [7].

The power coefficient of the rotor with a solidity of 0.375 is presented in Figure 5. It can be seen from Figure 5 that their maximum power coefficients increase with the reduction of the rotor solidity and they are 0.4 and 0.425 for the NACA0021 and the S1046, respectively. Their maximum operating tip speed ratios are increased to 2 and 2.25, respectively. After these TSRs, the power of the turbine with the NACA0021 case reduces sharply while the turbine with the S1046 section can continuously produce power and the reduction of the power occurs at a significantly slower rate. This small decrease after peak is the effect of the aerofoil thickness that contributes to a lower base drag at high, operating tip speed ratio. This characteristic is more pronounced when the solidity is lower [6].

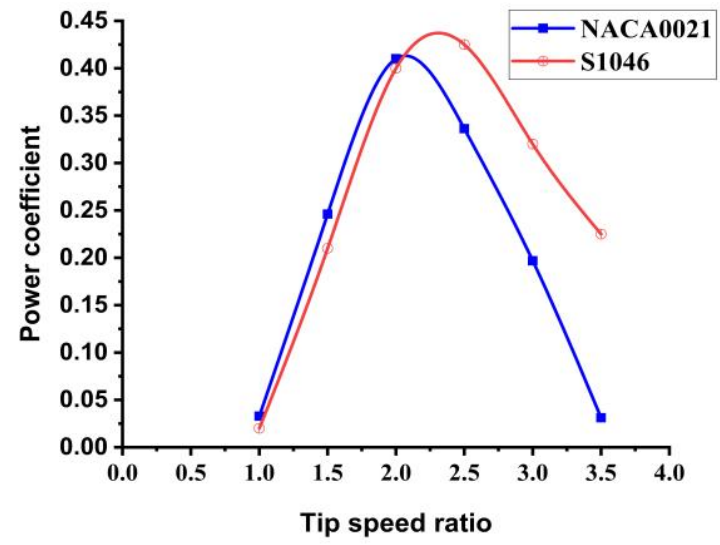

Fig. 5. Power coefficient at a wind speed $8 \mathrm{~m} / \mathrm{s}$ and a solidity 0.375

It is worth mentioning that the turbine with the NACA0021 section slightly outperform the turbine with the $\mathrm{S} 1046$ section at tip speed ratios lower than 2 . This is because, with the reduction of solidity, the induction effect that slows down the inflow velocity is reduced, causing both sections operate with a higher Reynolds number. With this high Reynolds number, the S1046 will be able to generate more lift and hence the total torque can be increased despite having a lower value of thickness.

In order to investigate their suitability for the urban application, the turbine performance at a wind speed of 6 $\mathrm{m} / \mathrm{s}$ are investigated and the results are presented in Figure 6 and 7. It can be seen from Figures 6 and 7 that the reduction of the wind speed reduces the power coefficient slightly for both cases. Reduction of the peak power are $4.9 \%$ and $3.09 \%$ for the NACA0021 and the S1046 sections, respectively.

For the S1046 case, this small reduction of power illustrates that the employment of a specifically designed aerofoil can improve the overall performance of the Darrieus turbine and the turbine will be able to operate with a wider range of operation. This is considered benefical when the turbine has to operate in the urban areas where the average wind speed is relatively low.

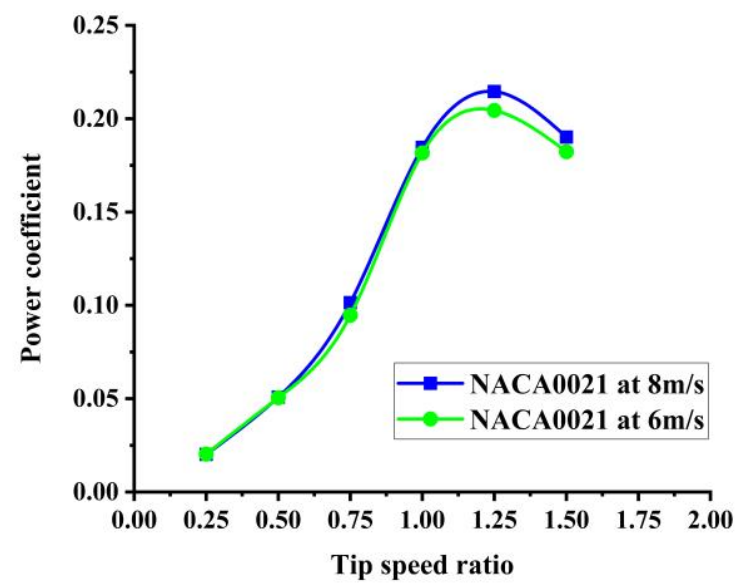

Fig. 6. Power coefficient of the NACA0021 at wind speeds of $6 \mathrm{~m} / \mathrm{s}$ and $8 \mathrm{~m} / \mathrm{s}$ 


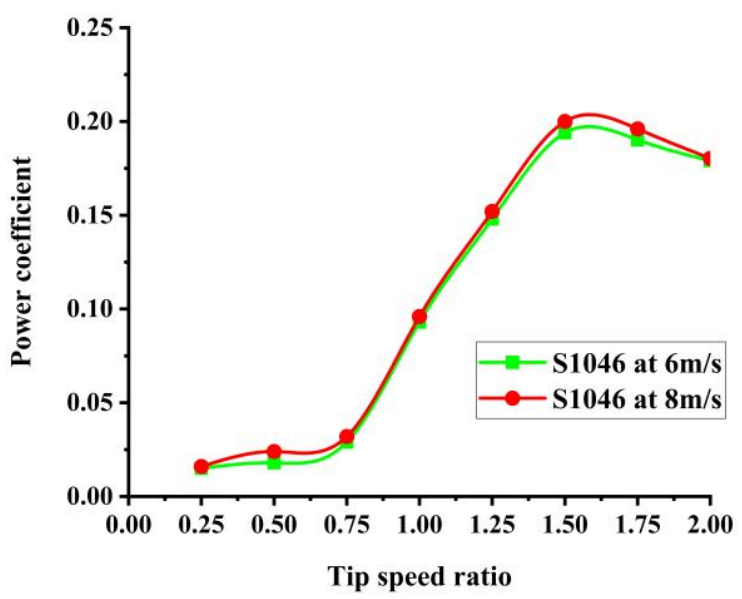

Fig. 7. Power coefficient of the $\mathrm{S} 1046$ at wind speeds of $6 \mathrm{~m} / \mathrm{s}$ and $8 \mathrm{~m} / \mathrm{s}$

\section{Conclusion}

Performance modelling of the Darrieus wind turbine was conducted in this paper. The validation of the modelling showed good agreement with the experimental results. The validated model was then employed to study the effects of the aerofoil shape and the rotor solidities. The results showed that the low solidity wind turbine in general operate at higher tip speed ratios and exhibits higher peak power which is around 0.4 compared to about 0.2 in the high solidity case.

The power coefficient of the turbine also depends upon the aerofoil shape. The employment of the conventional NACA0021 section shows good performance in the low tip speed ratio range. Nevertheless, the employment of the S1026 aerofoil section can significantly improve the turbine performance at low tip speed ratios especially for the low solidity rotor, which generally possesses a high power peak property. It is then recommended that the S1046 aerofoil section should be utilized with the turbine that has low solidity especially if the turbine is expected to operate with a wide range of tip speed ratios.

\section{References}

1. M. Kaya, M. Aksoy, F. Kose, Renewable Energy in Turkey: Potential, Current Status and Future Aspects, The International Journal of Engineering, 15, 1 (2017): 65-69

2. A. Bahaj, L. Byers, P. James, Urban Energy Generation: Influence of Micro Wind Turbine Output on Electricity Consumption in Building, Energy and Buildings, 39, 2 (2007): 154-165

3. Y. Wang, X. Sun, X. Dong, B. Zhu, D. Huang, Z. Zheng, Numerical Investigation on Aerodynamic Performance of a Novel Vertical Axis Wind Turbine with Adaptive Blades, Energy Conversion and Management, 108, (2016): 275-286
4. S. Worasinchai, Small Wind Turbine Starting Behaviour, PhD Thesis, Faculty of Engineering and Computing Sciences, The University of Durham, (2012)

5. H. Beri, Y. Yao, Effect of Camber Airfoil on Selfstarting of Vertical Axis Wind Turbine, Journal of Environmental Science and Technology, 4, 3 (2011): 302-312

6. M. Mohamed, Performance Investigation of H-rotor Darrieus Turbine with New Airfoil Shapes, Energy, 47, 1 (2012): 522-530

7. M. Castelli, E. Benini, Effect of blade thickness on Darrieus Vertical-Axis Wind turbine performance, The 2nd International Conference on Computer Modelling and Simulation, (2011)

8. S. Takahashi, J. Hamada, Y. Takashi, Numerical and Experimental Studies of Airfoils Suitable for Vertical Axis Wind Turbines and An Application of Wind-energy Collecting Structure for Higher Performance, Proceedings of the 19th National Symposium on Wind Engineering, (2006) 327-330.

9. F. Feng, S. Zhao, C. Qu, Y. Bai, Y. Zhang, Y. Li, Research on Aerodynamic Characteristics of Straight-Bladed Vertical Axis Wind Turbine with S Series Airfoils, International Journal of Rotating Machinery, (2018)

10. I. Hashem, M. Mohamed, Aerodynamic Performance Enhancements of H-rotor Darrieus Wind Turbine, Energy, 142, (2017), 531-545

11. M. Salam, V. Deshpande, S. Panday, N. Khan, M. Ali, Improvement in Aerodynamic Performance of NACA0021 Airfoil Using Moving Surface Boundary Layer: A computational study. AIP Conference Proceedings 2121, (2019).

12. M. Castelli, G. Grandi E. Benini, Numerical Analysis of the Performance of the DU91-W2-250 Airfoil for Straight-Bladed Vertical-Axis Wind Turbine Application, International Journal of Aerospace and Mechanical Engineering, 6, 3 (2012)

13. M. Elkhoury, T. Kiwata, E. Aoun, Experimental and Numerical Investigation of a Three-dimensional Vertical-axis Wind Turbine with Variable-pitch," J. Wind Eng. Ind. Aerodyn. 139, (2015), 111-123

14. P. Kozak, Effects of Unsteady Aerodynamics on Vertical-axis Wind Turbine Performance, M.S. Thesis, Illinois Institute of Technology, (2014)

15. H. Schlichting, H., Boundary Layer Theory, McGraw- Hill book company, New York, (1996)

16. F. Menter, Two-equation Eddy-viscosity Turbulence Models for Engineering Applications, American Institute of Aeronautics and Astronautics Journal, 32, 8 (1994), 1598-1605 